\title{
Century Identification and Recognition of Ancient Tamil Character Recognition
}

\author{
S.Rajakumar \\ Research scholar \\ Sathyabama University \\ Department of ECE \\ Chennai, India.
}

\author{
Dr.V.Subbiah Bharathi \\ Principal \\ DMI College of Engineering \\ Department of CSE \\ Chennai, India
}

\begin{abstract}
Recognition of ancient Tamil hand written characters from inscriptions is difficult. Ancient Tamil characters are different from current century's Tamil character. This paper concentrates on the century identification of ancient Tamil characters and converting them into current century's form using MATLAB. In this paper, a method for recognizing Tamil characters from stone inscriptions, called the contour-let transform, which has been recently introduced, is adopted. From previous research works, it's noticed that Wavelet transforms are not capable of reconstructing curved images perfectly. The contour-let transform offers a solution to remedy to this insufficiency. Contour-let transform is a $3 \mathrm{D}$ approach technique whereas wavelet transform is a $2 \mathrm{D}$ technique. The characters from the input image are recognized through clustering mechanism. Further the noise present in the image is removed by fuzzy median filters. Neural networks are employed to train the image and compare the data with the current century's character. Hence a more accurate recognition of Ancient Tamil characters from stone inscriptions is obtained.
\end{abstract}

\section{Keywords}

Contour-let transform, Wavelet transform, Fuzzy median filters, Neural network, Clustering.

\section{INTRODUCTION}

Prompt growth of technology and prevalent use of computers in the business and other areas, more and more organizations are converting their paper documents into electronic documents that can be processed by computers. Recognition of any hand written characters with respect to any language is difficult. Tamil, the native language of a southern state in India has several million speakers across the world and is an official language in countries such as Srilanka, Malaysia \& Singapore. Tamil script recognition differs from other language scripts in a few significant ways. As not every artifact can be re-documented because it is stolen or lost over time, so we have to process existing documentation. Before and after the invention of paper, writings were inscribed e.g. into stone, ceramic or metal. So we use image processing techniques to recognize the characters efficiently. In this paper, the image is processed such that its century is identified \& recognized in 3D based techniques.

\section{BLOCK DIAGRAM}

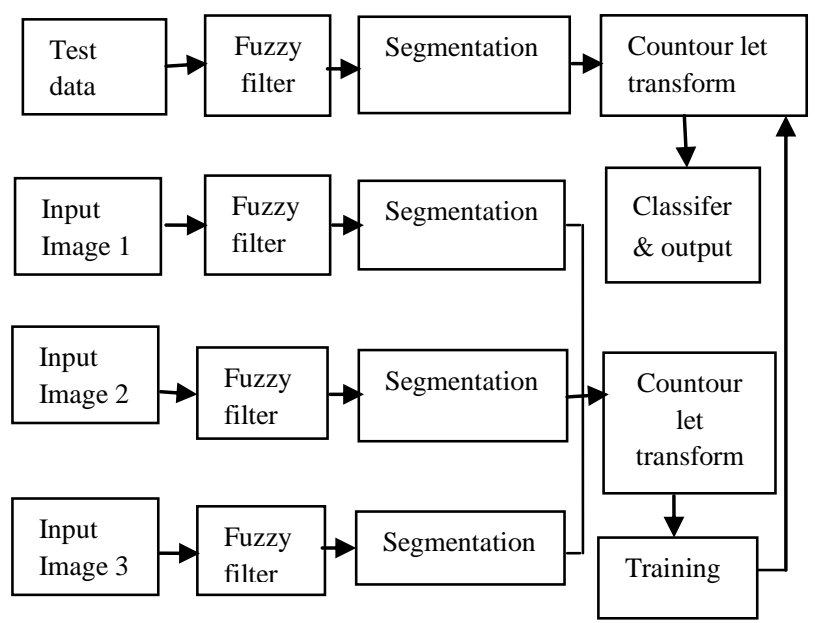

Fig 1: Block Diagram

\section{PREPROCESSING}

Preprocessing is the first step in the processing of character recognition. The input image is preprocessed for noise removal. The resultant image is checked for skewing. There are possibilities of image getting skewed with either left or right orientation. Here the image is first brightened and binarized.The function for skew detection checks for an angle of orientation \pm 15 degrees and if detected then a simple image rotation is carried out till the lines match with the true horizontal axis, which produces a skew corrected image. The exact skew angle is calculated by taking the average of all values within a specified range. The next process is smoothing. The task of smoothing is to remove unnecessary noise present in the image. Fuzzy median filter could be used. To reduce the effect of noise, the image is smoothened using a Gaussian filter. A Gaussian is an ideal filter in the sense that it reduces the magnitude of higher spatial frequencies in an image proportional to their frequencies. That is it reduces magnitude of higher frequencies more. The next step is thresholding. Thresholding is a nonlinear operation that converts a gray scale image into a binary image. The task of thresholding is to extract the foreground from the background. 


\subsection{Algorithm}

The input images of various ancient Tamil characters are collected from various century stone inscriptions.

$>$ The database is created by cropping the individual characters from the collected images.

$>$ The same character in different areas of the image is cropped and grouped under a single folder for training. More than 30 same characters are grouped under this folder.

$>$ For the purpose of displaying the current century characters, the current century Tamil characters are also collected, cropped \& grouped in a separate folder.

\subsection{Fuzzy Median Filter for Noise Removal}

$>$ Sort the obtained response values by ascending order, the middle most value is the median.

$>$ The absolute value is got by the difference of the surrounding values from its center value i.e., [(a11-a22), (a12-a22), (a33-a22) ].

$>$ From this set take the minimum of five values.

$>$ Now the value $\mathrm{d}=$ addition of min five,

$$
\begin{aligned}
& \text { If } \mathrm{d}<10 \text { - original pixel retains, } \\
& \text { If } 10<\mathrm{d}<30 \text { then } \mathrm{f}=(\mathrm{d}-10) /(30-10) \text { and } \\
& \text { Resultant pixel }=(1-\mathrm{f}) * \text { original }+\mathrm{f} * \text { median, } \\
& \text { If } \mathrm{d}>30 \text { - resultant pixel=median value. }
\end{aligned}
$$

Step1. Identify neighbor pixels, $\mathrm{x}(\mathrm{k})$ for the central pixel $\mathrm{x}$ of a window.

\section{Step2. Fuzzify $\mathrm{x}$ over the domain $\mathrm{X}$ with granules $L X i$}

Where $\mathrm{i}=1$ to $\mathrm{L}$ and their equivalent fuzzy sets can be expressed as $L X i=\int x \mu L X i(x) / x \quad \mid x \in X$

Where $X-$ center pixel value.

Step3. Fuzzify y over the domain $\mathrm{Y}$ with granules $L Y i$

Where $\mathrm{i}=1$ to $\mathrm{L}$ and their equivalent fuzzy sets can be expressed as $L Y i=\int y \mu L Y i(x) / x \quad \mid y \in Y$

Step4. Develop the fuzzy rule-base in the form of the fuzzy rules.

$$
R_{i}: \operatorname{IF} \bigcap_{k=1}^{8} x_{k}^{(i)} \text { is } L X_{k}^{(i)} \operatorname{THEN} y^{(i)} \text { is } L Y^{(i)} ; i=1 \text { to } N
$$

Where $X(k)$ - neighbours of center pixel value.

The implication is employed to interpret the meaning of a fuzzy rule $R i$ as a fuzzy relation $\widetilde{R_{l}}$. Then the compositional inference results the fuzzy rule-base as an equivalent fuzzy relation expressed as

$$
\tilde{R}=\bigcup_{i=1}^{n} \widetilde{R_{l}}
$$

Where $\widetilde{R}$ - Fuzzy rule

$R_{i^{-}}$Fuzzy relation
Step5. Fire the fuzzy rule-base of FMF.

a) Read $x^{*}$ as center pixel of current $3 \times 3$ window.

b) Identify corresponding neighbor pixels as $x_{k}^{*}$

c) Fuzzify $x_{k}^{*}$

$$
\begin{gathered}
\forall X_{k}: \mu_{L X^{*}\left(x_{k}^{*}\right)}==\left\{\begin{array}{l}
1 ; \text { if } x=x_{k}^{*} \\
0 ; \text { itherwise }
\end{array}\right. \\
L \widetilde{X_{k}^{*}}=\int_{x} \mu_{L X^{*}\left(x_{k}^{*}\right)} / x
\end{gathered}
$$

d) Compute the fuzzified output of FMF.

$$
\mathrm{CL} \widetilde{Y^{*}}=\mathrm{L} \widetilde{X^{*}} R=\int_{y} \mu_{C L Y}(y) / y^{\circ}
$$

e) Defuzzify $C L \widetilde{Y^{*}}$ using Center of Gravity method.

$y^{*}=\frac{\int_{y} y \cdot \mu_{C L Y}(y) d y}{\int_{y} \mu_{C L Y}(y) d y}$

Step6. Restore the corrupt pixel

$$
x^{*}=y^{*}
$$

Step7. Repeat the steps 1 to 6 to restore all the corrupt pixels of noisy image.

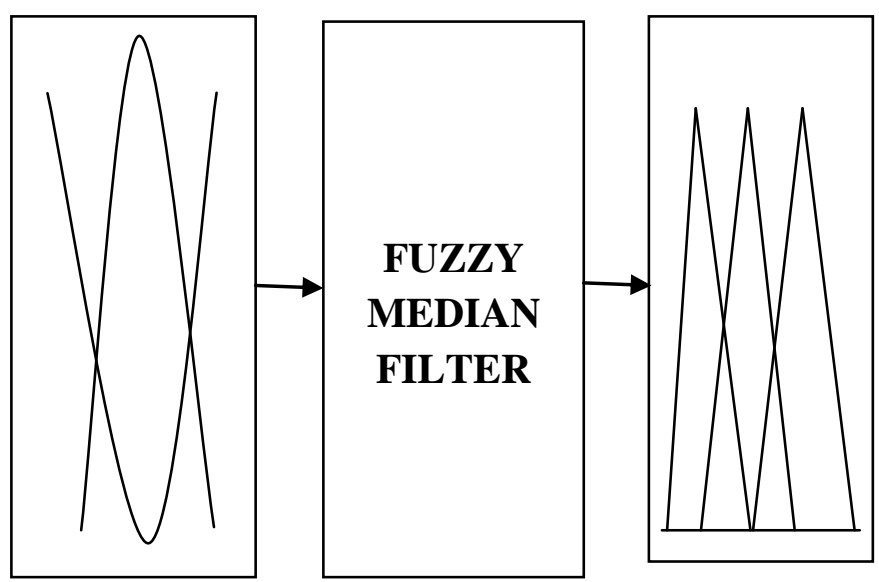

Fig 2: Graph for Fuzzy median filter

\section{SEGMENTATION BY CLUSTERING}

Whatever we need to group is considered as tokens (pixels, points, Surface elements, etc,). In Top down segmentation the tokens belongs together because they lie on the same object. In Bottom up segmentation the tokens belong together because they are locally coherent. These two are not mutually exclusive. Initially, given $\mathrm{K}$ categories and , $\mathrm{N}$ points in feature space, Pick K points randomly; these are initial cluster centers (means) $\mathrm{m} 1, \mathrm{~m} 2, \mathrm{~m} 3, \ldots, \mathrm{mK}$. Repeat the following:

1. Assign each of the $\mathrm{N}$ points, $\mathrm{xi}$, to clusters by nearest $\mathrm{mi}$ (make sure no cluster is empty).

2. Recomputed the Mean mi of each cluster from its member Points. 
3. If no mean has changed, then you stop the process and do the clustering using the following method

$$
\sum_{\mathrm{i} \epsilon \text { clusters }} \sum_{\mathrm{j}=\text { elements of ith cluster }}\left\|\mathrm{x}_{\mathrm{i}}-\mu_{\mathrm{i}}\right\|^{2}
$$

4.Minimizing squared distances to the center implies that the center is at the mean

$$
\begin{gathered}
\frac{\partial \mathrm{e}}{\partial \mathrm{m}_{\mathrm{k}}}=\sum_{\mathrm{j} ; \mathrm{c}_{\mathrm{j}}=\mathrm{k}}-2\left(\mathrm{x}_{\mathrm{j}}-\mathrm{m}_{\mathrm{k}}\right)=0 \\
\frac{\sum_{\mathrm{j} ; \mathrm{c}_{\mathrm{j}}=\mathrm{k}} \mathrm{x}_{\mathrm{j}}}{\sum_{\mathrm{j} ; \mathrm{c}_{\mathrm{j}}=\mathrm{k}} 1}=\frac{1}{\mathrm{n}_{\mathrm{k}}} \sum_{\mathrm{j} ; \mathrm{c}_{\mathrm{j}}=\mathrm{k}} \mathrm{x}_{\mathrm{j}}
\end{gathered}
$$

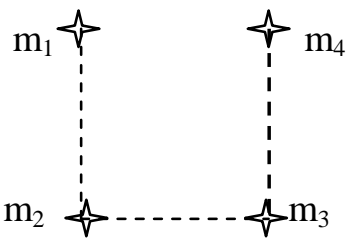

Fig 3. Selection of mean point

\section{RECOGNITION}

The term neural network was traditionally used to refer to a network or circuit of biological neurons. The modern usage of the term often refers to artificial neural networks, which are composed of artificial neurons or nodes. Biological are made up of real biological neurons that are connected or functionally related in the peripheral nervous system or the central. In the field of neuroscience, they are often identified as groups of neurons that perform a specific physiological function in laboratory analysis. Artificial neural networks may either be used to gain an understanding of biological neural networks, or for solving artificial intelligence problems without necessarily creating a model of a real biological system. The real, biological nervous system is highly complex and includes some features that may seem superfluous based on an understanding of artificial networks.

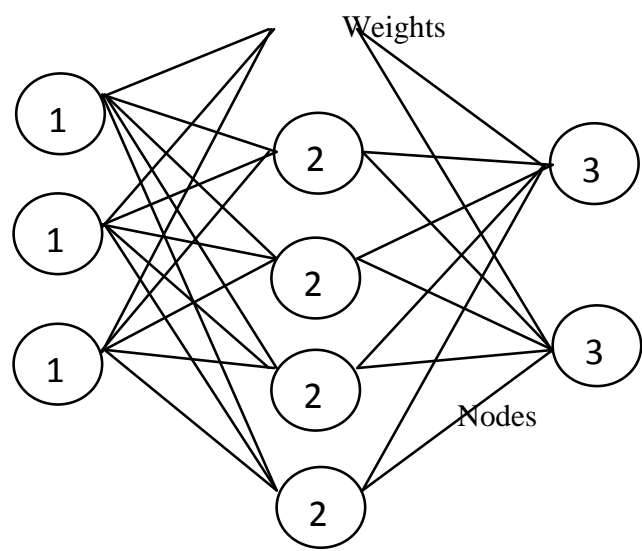

Fig4. Representation of a neural network

In the above figure the leftmost nodes assign initial weights randomly and train continuously. The weights are added and stored in the centremost nodes. Several nodes are generated and then fall between $o$ and 1 based on threshold generated. Now the final weights are compared with the input and the process is reversed to minimise the error. Finally based on the weights the database is compared with the test data and if the weight matches, the corresponding character is displayed. The same process takes place while training the single character which is cut from the input image to find the features of the corresponding test data. Thus neural network reduces the manual training and recognises the output easily. The basic requirement of neural network is, it needs necessary data to train more accurately. The weights will be different for different inputs which we feed.

\section{RESULTS}

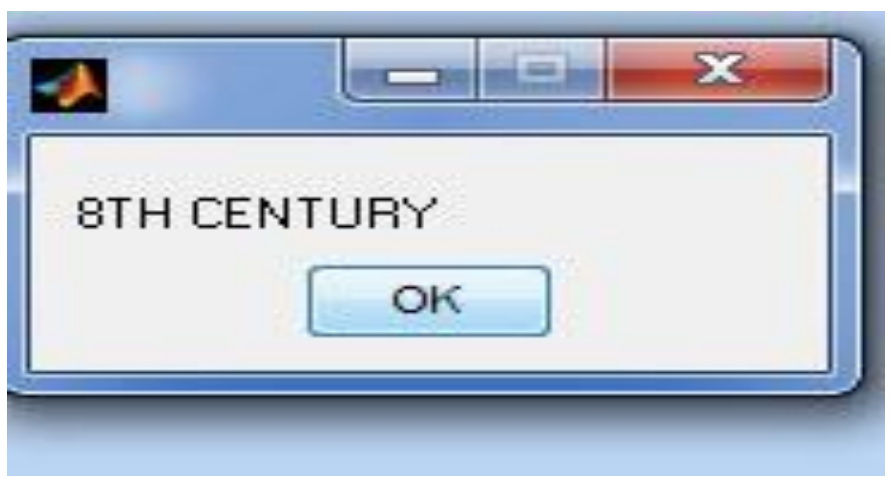

Fig5.Century Identification

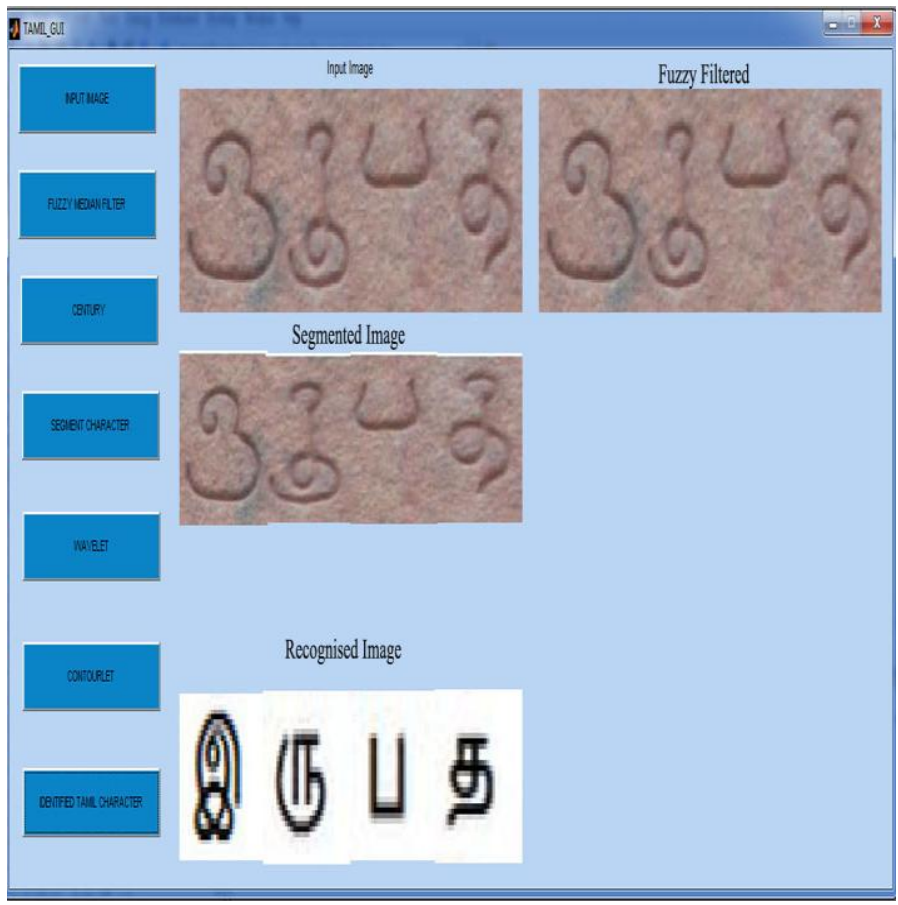

Fig6. Ancient Tamil Character Recognition 


\section{CONCLUSION}

In this paper, we presented a new contour let-based method on offline text-independent ancient Tamil handwriting identification. Compared with methods via 2-D wavelet, contour let-based method achieves a higher accuracy because contour let transform has capacity to capture comparatively richer directional information, which is important to represent the writing style of handwriting. Since there is no existed handwriting database available to us, we have to build up a small handwriting database ourselves, as leads to the small capacity of our experimental database. To make our experiment result more convincing, we plan to enlarge our database in future work.

\section{REFERENCES}

[1] A Contour let-based Method for Writer Identification by 1 . Zhenyu He, 2.Yuan Yan Tang and 3.Xinge you, 2008

[2] On-line and Off-line Handwriting Recognition: A Comprehensive Survey".IEEE Trans. on Pattern Analysis and Machine Intelligence, 22(1):63-84, 2006 by R. Plamondon and S. Srihari

[3] Y.Lecun,L.Bottou,Y.Bengio,P.Haffner, “Gradient based learning applied to document recognition" Proceedings of the IEEE, vol. 86, no.11, IEEE, pp. 2278- 2324, USA, 1998.

[4] Robust Fuzzy Median Filter for Impulse Noise Reduction of Gray Scale Images by Jagadish H. Pujar

[5] Artificial Neural Networks by Torsten Reil, 2004.

[6] H.E.S.Said, T.N.Tan, K.D.Baker, "Personal identification based on handwriting," Pattern Recognition, Vol 33, No. 1, pp. 149- 160, 2000.

[7] E.N.Zois, V.Anastassopousls, "Fusion of correlated decisions for writer verification," Pattern Recognition, Vol 33, No. 10, pp. 1821-1829, 1999

[8] D. D.Y. Po, M. N. Do, "Directional multiscale modeling of images using the contourlet transform"

[9] M. N. Do and M. Vetterli, "Wavelet-based texture retrieval using generalized Gaussian density and Kullback- Leibler distance".

[10] M. N. Do, M. Vetterli, "The contourlet transform: An efficient directional multiresolution image representation," 